\title{
Dutch-Book Arguments against Using Conditional Probabilities for Conditional Bets
}

\author{
Keith Hutchison \\ School of Historical and Philosophical Studies, University of Melbourne, Parkville, Australia \\ Email: keithrh@gmail.com
}

Received April 22 $2^{\text {nd }}, 2012$; revised May $24^{\text {th }}, 2012$; accepted June $10^{\text {th }}, 2012$

\begin{abstract}
We consider here an important family of conditional bets, those that proceed to settlement if and only if some agreed evidence is received that a condition has been met. Despite an opinion widespread in the literature, we observe that when the evidence is strong enough to generate certainty as to whether the condition has been met or not, using traditional conditional probabilities for such bets will not preserve a gambler from having a synchronic Dutch Book imposed upon him. On the contrary (I show) the gambler can be Dutch-Booked if his betting ratios ever depart from a rather different probability, one that involves the probability of the agreed evidence being provided. We note furthermore that this same alternative probability assessment is necessary if the evidence is weaker (i.e. if it fails to provide knowledge whether or not the condition has been met). By contrast, some of the (rather different) probability assessments proposed by Jeffrey, precisely for such situations, still expose the gambler to a Dutch-Book.
\end{abstract}

Keywords: Probability; Conditioning; Dutch-Book; Conditional Probability; Bayesianism; Jeffrey Conditioning

\section{Introduction}

My core task below is to refute a common belief about conditional probabilities, the claim that such probability assessments preserve a gambler from having a Dutch-Book imposed upon him when he makes an important type of conditional bet, one that proceeds to settlement if and only if the participating gamblers find out that some agreed condition has been met. The discussion below, accordingly, is set in the standard DutchBook context: i.e. two gamblers (Peter and Mary say) are betting against each other on the outcomes of some chance-process, with Peter setting the odds through his assessments of the probability $p(Z)$ of each outcome $Z$, and Mary setting the bets.

It is well-known that if Peter's odds are "coherent" (i.e. obey the basic laws of probability), Mary cannot offer Peter a book composed of simple bets that will result in a net gain for Mary no matter which of the possible outcomes $Z$ eventuates. For the purposes of the discussion here, we shall call such a book "Dutch" (and we will refer to the gains that it provides Mary as "sure", or "guaranteed" etc.) ${ }^{1}$.

The existence of such a Dutch-Book is often taken as evidence that Peter's odds are irrational, but that judgment is a little hasty. For in many circumstances, Peter can find himself guaranteeing Mary a profit, because of simple ignorance, as opposed to some blemish of reasoning - as becomes fleetingly important at the very end of our discussion. Though quite familiar, this possibility is often blurred in the literature, so we need to distinguish two types of Dutch-Books: "weak" ones, those that result from ignorance, and which cannot accordingly

\footnotetext{
${ }^{1}$ We will not (as sometimes suggested) require a Dutch-Book to guarantee a profit whatever happens, for that is exceptionally vague, and far too broad. So if gamblers are betting on the toss of a normal coin, identification of a Dutch-Book will not require us to show that a profit would occur even if the head on the coin mysteriously turned into a tail during the toss, or worse, if the world was destroyed before the coin landed.
}

be anticipated by Peter; and "stronger" ones-where Peter possesses enough information to perceive his odds will allow a guaranteed loss to be imposed upon him. Not all stronger Dutch-Books are, however, evidence of irrationality either, but we can avoid confronting this issue. Except for the cases briefly mentioned in my search for a converse below, all the DutchBooks used in the argumentation below will be of the "irrational" type.

It is widely recognized that Peter's safety-net can be extended beyond simple bets, to an important category of conditional bets. If a bet on $X$ is to proceed to settlement when and only when the outcome $Y$ has been achieved, Peter is protected if he accepts bets based on the "conditional probability" $p(X \& Y) / p(Y)$, abbreviated here as $p(X / Y)^{2}$. Let us call such bets "ontic" conditional bets, because they proceed if and only if the outcome at issue has come into existence. We need to give them a name, for our aim here is to point out that the familiar safety-net does not extend to a wider class of conditional bets.

If indeed we attend to the distinction between a proposition's

\footnotetext{
${ }^{2} \mathrm{~A}$ number of theorists do not treat $p(X / Y)$ as an abbreviation, but as a primitive notion, "the probability of $X$ given $Y$ ". For our present purposes, this approach is too ambiguous however, for "given $Y$ " can mean either "given the truth of $Y$ " or "given a knowledge of $Y$ ". An ongoing theme of this paper could then be rephrased as the pair of claims: a) that these two quantities are different, and $b)$ that $p(X / Y)$ in the first sense is not suitable for epistemic conditional bets. The relationship between such conditional bets and the second, epistemic, sense of $p(X / Y)$ is more complicated: for "diachronic" books (those involving probabilities that change in the course of time), become involved. The analysis thus expands to hazier "kinematic" issues outside the deliberately focussed scope of this essay- the synchronic context set by the literature I seek to refute. Indeed, a common way of interpreting conditional probabilities is to presume (in the example used below) they refer to new assessments of probability that Peter adopts around the time $\tau+4$ (in time-line below). In virtually all the discussion here, probability assessments are made at the one time $(\tau-2)$, and the same applies to stakes $(\tau-1)$.
} 
being ascertained and its being true ${ }^{3}$, we will observe that such ontic bets are distinct from "epistemic" ones-which proceed if and only if the outcome $Y$ has been detected. Epistemic conditional bets are far more realistic than the ontic ones, for ordinary gamblers cannot be expected to settle a bet if its precondition is not known to be true - even if it is in fact true. They are, too, the sort of bets that specially interest Bayesians, for the core of Bayesianism is analysis of the effects of new information. In many artificial situations of course (especially those typical of the text-book, casino, or appeals to god), the truth or falsity of the applicable condition is always ascertained, so the distinction between the two classes of bet fades. But this is not generally true.

There is a presumption throughout the literature that the argument which preserves a gambler from being Dutch-Booked via ontic conditional bets, covers the epistemic ones as well ${ }^{4}$. But that is definitely not true. Demonstrating this fact is the central task undertaken in the present essay - which thus supplements an earlier piece of mine (Hutchison, 1999), that demonstrated the inadequacy of conditional probability as a procedure for updating probabilities in the light of supplementary evidence.

The core of the present argument is the simple counter-example set out with pedantic care below. It quickly shows that various Dutch-Books (of the irrational kind) can be set up against Peter, if Peter adopts the strategy of betting on an outcome $X$ at the rate $p(X \& Y) / p(Y)$ when bets are settled if and only if the outcome $Y$ is detected. Peter, indeed, will not be able to rely on a calculation that (like the traditional formula) uses only the probabilities he attributes to the outcomes of the chance-process under observation. For protection against DutchBooks, he needs to supplement these probabilities with something else: those associated with the gamblers' finding out that

\footnotetext{
${ }^{3}$ As evidenced by the citations in $\mathrm{n} .4$ below, there is remarkable little attention to this distinction in the probability literature. It is, fleetingly, recognised as being a problem in the subjective interpretation, but seemingly tolerated. Hacking, 1967: pp. 316, 324 treats it as a "trifling idealization" typical of those made in philosophical analysis; while Weatherson, 2003 and Harman, 1983 note that the blurring creates an affinity with intuitionist logic, without however observing the dramatically disruptive consequences within more standard logics. Howson \& Urbach, 2006: p. 54 avoid the issue by presuming (in one particularly sensitive context) that bets are settled by an "omniscient oracle".

${ }^{4}$ Because the literature on probability is reluctant to distinguish the two types of bets (and more generally, to accommodate the distinction between truth and ascertainment), we know of no overt declaration that the one argument covers both cases. But it is routine to find an equivocation in the interpretation of the result at issue in the context of discussions of the Dutch-Book defence of conditionalization. See, e.g. De Finetti, 1974-1975, v.1: p. 135 ("if $H$ does not turn out to be true"; "if I know $H$ is true"; brief cryptic reference to Dutch-Book); Gillies, 2000: pp. 36-37 (where conditional probabilities are introduced as if they refer to what is ascertained) and p. 62 (where the Ramsey-De Finetti theorem is stated in terms of truth-with bets being called off if a condition "does not occur" - then defended via a Dutch-Book argument); Talbott, 2008: p. 2 (where conditionalization is characterised epistemically) and p. 3 (where the Dutch-Book argument is phrased in terms of a bet called off if a condition is not true); Howson, 1977: p. 63 (middle paragraph, where the conclusion of the Dutch-Book argument is summed up in terms of both truth and verification); Teller, 1973: p. 220 (where the problem is phrased in terms of ascertainment), p. 222 (where the reference class singled out by ascertainment is identified with that provided by truth), p. 224 (where the circumstances in which bets are called off is characterised via truth); Skyrms, 1987: p. 2 (where the problem is phrased in terms of ascertainment), p. 3 (where the circumstances in which bets are called off is characterised via truth).
}

the outcome was $Y$.

To establish the alternative calculation that Peter needs to use, our discussion (following the initial presentation of the main counter-example) becomes distinctly more complicated, but only superficially so, in that we have to juggle a multiplicity of probabilities. We then extend this key result even further, showing that our alternative formula applies when bets are made conditional upon the receipt of evidence for $Y$, and that evidence falls short of providing knowledge that the outcome $Y$ has been achieved. This situation is sometimes supposed to be covered by Jeffrey's extended rule of conditioning (see n. 9), but we show that this rule advises Peter to use rather different betting ratios, and that these do not suffice to exclude a Dutch-Book.

As befits the fact that I am arguing against a received claim, my focus will be almost exclusively on the "synchronic" books used in the literature that I target, those in which all bets are placed at the one time. Diachronic books (i.e. those composed of bets placed at different times) generate dramatic complications for the Dutch-Book approach-see e.g. n. 5-and accordingly are given only passing mention below, though I have discussed the inadequacy of conditional probability in their context elsewhere (see Hutchison, 1999).

\section{The Betting Scenario for the Main Example}

To make the case, we consider a very simple urn model. (Many readers will recognize this to be an adaption of Bertrand's box paradox, but that fact is not important to following the analysis below). We will be supposing that (at some time we shall call $\tau$ ) an honest (etc.) coordinator Paul places three unbiased coins in an urn: one normal coin (with a head and a tail face); one with two head faces; and one with two tail faces. Later, at time $\tau+1$, Paul randomly selects one of these coins from the urn, and Peter and Mary bet against each other on (inter alia) the various outcomes of this draw. These bets can be simple; or conditional upon information released by Paul.

For after he makes the draw, Paul is to release information to Paul and Mary, via a two-stage process (summarized in the time-line depicted below). The second stage is rather trite and takes place at time $\tau+5$, when Paul reveals full details of the selected coin, so that bets can be settled (at $\tau+6$ ). The first stage is far more significant here, for this takes place earlier (at time $\tau+$ 3 ), when Paul provides the partial information about the draw that allows epistemic conditional betting. Paul inspects the coin that has been drawn, and reveals something about it via procedures specified in the individual examples below.

We suppose that Peter and Mary have both understood all Paul's proposed actions since well before they were actually carried out, and that each of them also understands that the other possesses the same comprehension of the processes. So all that they later discover is the results of those activities. To avoid all irrelevant confusion about the temporal sequence of events, we suppose that this understanding of the process was in place at time $\tau-3$, and we shall be irritatingly careful to stress the timings of all later activity (as summarized in our time-line, Figure 1). In the end, these timings are not very important, but it is vital that there be no room for confusion about them.

It may not be important here that Mary know that Peter shares her understanding of the betting process, but Peter's realization that Mary understands what is going on does seem vital to the logic - because this, in the end, is what makes the Dutch-Books below evidence of Peter's irrationality in agreeing to bet on standard conditional probabilities. For it means that Peter has enough 


\section{K. HUTCHISON}

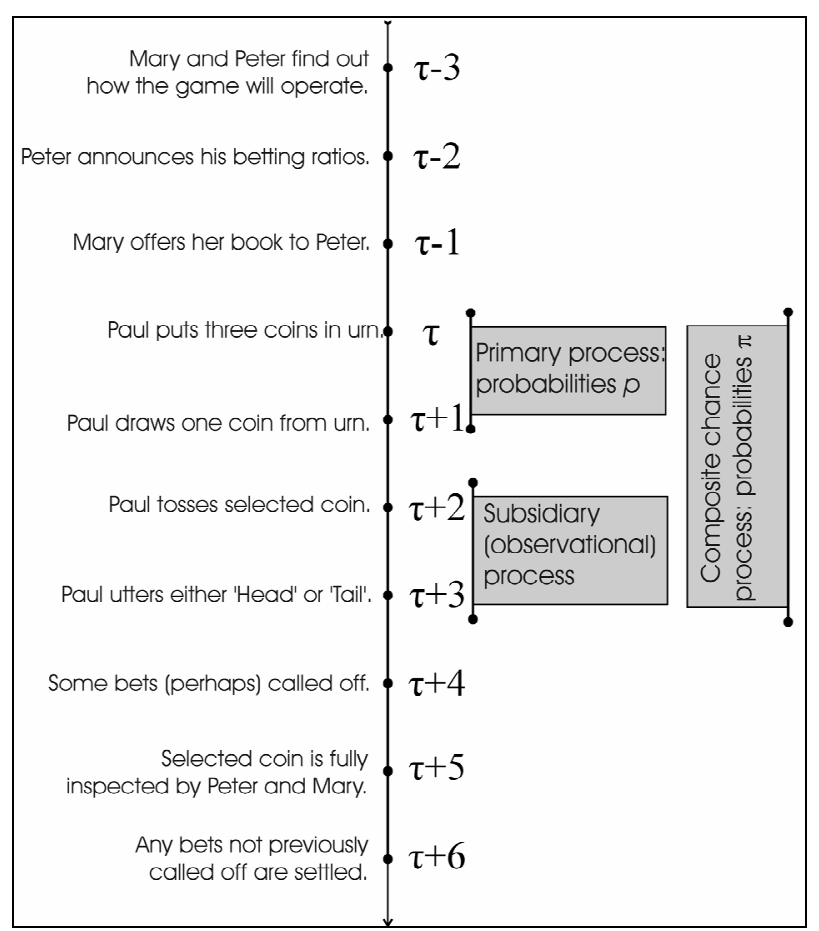

Figure 1.

TIME-LINE (left): the precise sequence of events in our counter-examples: placing bets; drawing coin; releasing information; settling bets. The two separate chance-processes involved (right).

information to realize that Mary has access to the various profitable algorithms articulated below ${ }^{5}$. Reason alone can then tell Peter that in allowing Mary to set the bets, he has in effect agreed to give Mary full access to his bank account. She can take from him as much money as she wants, and (granted she is able to do the requisite arithmetic etc.) is restrained by nothing more than her willingness to act.

After he has acquired this understanding of what was going to happen, but still before the chance-process actually begins, Peter announces (at time $\tau-2$ say) the probabilities that he was willing to bet on here; and bets are subsequently offered by Mary (at time $\tau-1$ ).

Amongst the probabilities announced by Peter at time $\tau-2$, are his degrees-of-belief in the various outcomes $Z$ of the draw that Paul is soon to make. These generate a very simple probability function $p(Z)$, which (in addition to 0 and 1 ) takes the following values:

$\begin{aligned} 1 / 3= & p(H H) \text { for the selected coin having two } \\ & \text { heads (which we call outcome " } H H^{\prime} \text { ); } \\ 1 / 3= & p(T T) \text { for the selected coin having two tails } \\ & (\text { outcome "TT"); }\end{aligned}$

${ }_{5}^{5}$ Irrationality definitely requires more than a) Peter having enough understanding to be able to see that a Dutch-Book exists, or b) that Mary be able to see the same thing. For if we briefly consider bets placed at different times, it is easy to show that a diachronic Dutch-Book exists whenever Peter changes his betting ratios. Yet rationality often requires him to do this (typically, when new evidence arises). Mary can then stumble upon a Dutch-Book, but she has no algorithm to construct it. For she cannot know the appropriate initial bet until she also knows what change Peter is going to make to his betting ratio.

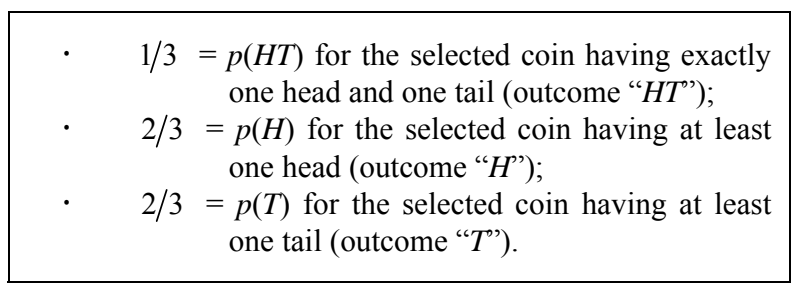

\section{Principal Counter-Example}

To generate our first pool of conditional bets, we suppose that Paul is to release his partial information, by uttering (at time $\tau+3$ ) precisely one word, either "Head" or "Tail". Paul chooses between these two utterances by tossing (at time $\tau+2$ ) the coin previously selected (at time $\tau+1$ ). If this coin comes up heads at time $\tau+2$, Paul says "Head" at time $\tau+3$, but if it comes up tails he says "Tail". Since Peter has understood (since time $\tau-3$ ) that this is how Paul was going to behave, Peter allocates (at time $\tau-2$ ) the probability $1 / 2$ to each utterance. So (at time $\tau-2)$ Peter declares himself ready to bet (at time $\tau$ 1) on either utterance at this rate.

It is important to note that Paul's utterance emerges from a subsidiary-observational-chance-process that occurs between times $\tau+2$ and $\tau+3$, distinctly after the primary chance-process that is the nominal focus of the betting, the one that occurs between times $\tau$ and $\tau+1$. As reflects the fact that the information comes from a very different chance-process, the probability $(1 / 2)$ that Peter allocates to finding out the selected coin has at least one tail is less than the probability $(2 / 3)$ he allocates to its being true that it has at least one tail. Peter is clearly right here $^{6}$, and furthermore, this divergence between the two probabilities is at the heart of the matter at hand: bets that refer to what is known (as opposed to what is true) need to allow for the supplementary probabilities allocated to the outcomes of the observational chance-process.

To see this, we now enquire what fair betting-ratios Peter should endorse for bets on $H H, H T$, and $T T$, if such bets are conditional, and only proceed (beyond time $\tau+4$ ) when Peter and Mary have found out (at time $\tau+3$ ) that the draw produced at least one tail? ${ }^{7}$ Obviously, they acquire this knowledge if and only if Paul says "Tail" (at time $\tau+3$ ) (If this happens, bets on $H H$ will not, of course, proceed).

If Peter recognizes the affinity between our betting scenario and the literature on Bertrand's box paradox, he could well be guided by that literature to assess the probabilities to be used for his conditional bets on $H T$ and $T T$ as $1 / 3$ and $3 / 2$. But he could instead be guided by rather different literature, an unsatisfactory literature which clashes with the box literature, and the literature which is our prime target here. That is the impor-

\footnotetext{
${ }^{6}$ Since this divergence plays a critical role below, it might seem that a radically subjectivist Peter can avoid our Dutch-Book by insisting that his degree-of-belief in the "Tail"-utterance is actually somewhat higher. But then he exposes himself to other Dutch-Books. For if his degree-of-belief in the "Head"-utterance does not drop, he can be Dutch-Booked on the "Head" and "Tail" probabilities alone. And when it does drop, he falls prey to a Dutch-Book identical to that set out below, but with Heads and Tails interchanged.

${ }^{7}$ More carefully: What fair betting-ratios should Peter endorse (back at time $\tau-2$ ) for bets with Mary (at time $\tau-1$ ) on the three possible outcomes of the original draw (viz.: HH, HT, TT) - the outcomes that get revealed in a full inspection (at time $\tau+5$ ) of the selected coin-if such bets are now conditional, and only proceed (beyond $\tau+4$ ) when Peter and Mary find out (at time $\tau+3$ ) that the draw at time $\tau+1$ produced at least one tail?
} 
tant body of writings (as sampled in n. 4) which assures him that using conditional probabilities is appropriate here. He would then accept $p(X \& T) / p(T)[\equiv p(X / T)]$ as a fair basis for a bet on any outcome $X$ that is conditional upon the ascertainment of the outcome $T$. Such probabilities are easy to evaluate and in particular $p(T T / T)$ is just $p(T T \& T) / p(T)=p(T T) / p(T)=\frac{1}{3} / \frac{3}{2}$ $=1 / 2$.

These probabilities do not however protect Peter from being Dutch-Booked. For suppose that Mary sets up the following book:

Bet 1: Peter bets $\$ 4$ in favour of the outcome TT.

i.e. he pays Mary $\$ 4$, and in return:

- Mary pays him $\$ 12$ if (at time $\tau+6$ ), the $T T$ coin is found to have been drawn, for Peter's fair betting ratio here is $p(T T)=1 / 3$.

- $\quad$ She pays him nothing otherwise.

Bet 2: Mary bets $\$ 6$ in favour of the draw producing exactly two tails, but conditionally on at least one tail (outcome $T$ ) being revealed (i.e. conditionally on Paul uttering "Tail" at time $\tau+3)$.

i.e. she pays Peter $\$ 6$, and in return:

- Peter pays her $\$ 12$ (at time $\tau+6$ ) if two tails are found (at time $\tau+5$ ) (for if the coin has two tails Paul must utter "Tail" so the bet would not have been cancelled, and Peter's fair betting ratio here is, by hypothesis, 1/2).

- Peter pays her nothing if Paul utters "Tail" but a head and tail are found at time $\tau+5$.

Peter refunds her \$6 if Paul does not utter "Tail”.

Bet 3: Mary bets $\$ 3$ in favour of the "Tail" utterance.

i.e. she pays Peter $\$ 3$, and in return:

- Peter pays her $\$ 6$ (at time $\tau+6$ ) if Paul (at time $\tau+$ 3 ) utters "Tail" (for Peter's fair betting ratio here is $1 / 2)$.

- Peter pays her nothing if Paul utters "Head".

In so far as he has set the odds for these three bets, Peter claims to regard each of them as individually fair. Yet if he accepts the whole book, Mary will make a profit of $\$ 1$, no matter what outcome was produced in the original draw, or what utterance Paul makes at $\tau+3$. This is clear in the tabulation of her earnings set out in Table 1.

A little reflection will quickly indicate what has gone "wrong" here: Paul's "Tail"-message does not indicate that the initial draw has produced a coin with a tail every time that it does produce such a coin. There is indeed a $50 \%$ chance that the coin with one head and one tail will produce the "Head"-message.

Given the circumstances which produce the "Tail"-message, Peter should actually adopt a probability of $2 / 3$ (rather than $1 / 2$ )

Table 1.

Calculation of Mary's winnings: Principal counter-example.

\begin{tabular}{cccccccc}
\hline & \multicolumn{2}{c}{$\begin{array}{c}\text { Bet 1: TT } \\
(1 / 3)\end{array}$} & \multicolumn{2}{c}{$\begin{array}{c}\text { Bet 2: TT/“Tail” } \\
(1 / 2)\end{array}$} & \multicolumn{2}{c}{$\begin{array}{c}\text { Bet 3: “Tail” } \\
(1 / 2)\end{array}$} \\
\hline Outcome & Loss & Gain & Loss & Gain & Loss & Gain & Net Gain \\
\hline$H H$ & $\$ 0$ & $\$ 4$ & $\$ 6$ & $\$ 6$ & $\$ 3$ & $\$ 0$ & $\$ 1$ \\
$T T$ & $\$ 12$ & $\$ 4$ & $\$ 6$ & $\$ 12$ & $\$ 3$ & $\$ 6$ & $\$ 1$ \\
HT\&"Tail” & $\$ 0$ & $\$ 4$ & $\$ 6$ & $\$ 0$ & $\$ 3$ & $\$ 6$ & $\$ 1$ \\
HT\&"Head" & $\$ 0$ & $\$ 4$ & $\$ 6$ & $\$ 6$ & $\$ 3$ & $\$ 0$ & $\$ 1$ \\
\hline
\end{tabular}

for the conditional bet on $T T$ - the probability suggested by the box paradox literature. This $2 / 3$ is still a conditional probability, but it is very different from that recommended in the Dutch-Book literature, which endorses betting ratios derived exclusively from the probabilities $p(Z)$ of outcomes $Z$ of the pri mary chance-process. The safe probability, $2 / 3$, is rather $\pi(T T /$ "Tail" uttered) - where $\pi$ is the extension of $p$ that covers the composite chance process that stretches from time $\tau$ to $\tau+3$, the primary process plus its epistemic coda ${ }^{8}$. If Peter used this improved valuation, he would pay Mary only $\$ 9$ if two tails were confirmed, so she would then makes a net loss of $\$ 2$ if the coin had two tails. i.e. Peter would no longer be Dutch-Booked.

It might seem that this situation can be rectified by disallowing the epistemic protocol specified in this counter-example, the procedure followed by Paul in deciding whether to utter "Head" or "Tail". But that is thoroughly unsatisfactory-for two quite different reasons. Firstly, it amounts to an admission that conditional probabilities cannot handle thoroughly reasonable epistemic behavior: it in effect concedes the very point that we are trying to establish. Secondly, it does not solve the problem.

\section{Subsidiary Counter-Example}

For suppose we insist that Paul investigate the outcome of the initial draw differently, indeed so thoroughly as to utter "Tail" if and only if at least one side of the selected coin possessed a tail, and now ask that Peter again bet on the conditional probabilities. Peter will not then be Dutch-Booked if he buys the book we proposed for Mary in the principal counterexample above. For Peter will now estimate the probability of Paul uttering "Tail" to have increased to $2 / 3$, so some of Mary's income will be reduced. The $\$ 6$ she wins on bet 3 when Paul says "Tail", will drop to $\$ 4.50$, and the $\$ 1.50$ reduction here will wipe out her profit, and give her a 50c loss instead.

But Mary will now be able to offer a different book, that shown by Table 2 to be Dutch, and consisting of just two bets, one simple and the other conditional:

Bet 1: Mary bets $\$ 2$ in favor of the coin having at least one tail. i.e. she pays Peter $\$ 2$, and in return:

- Peter pays her $\$ 3$ whenever the coin is $T T$ or $H T$, (for Peter assesses the probability of $T$ as $2 / 3$ ).

Peter pays her nothing if the coin is $\mathrm{HH}$.

Bet 2: Mary bets $\$ 3$ on both sides of the coin being a head, but conditionally on at least one head (outcome $H$ ) being revealed, (i.e. conditionally on Paul uttering "Head" at time $\tau$ $+3)$.

i.e. she pays Peter $\$ 3$, and in return:

- Peter pays her \$6 if Paul utters "Head", (for by hypothesis, Peter is ready to bet on the conditional probability $p(H H / H)(=1 / 2)$ here, and by hypothesis also, Paul only utters "Head" when there is no tail).

Peter refunds the $\$ 3$ if Paul utters "Tail".

\footnotetext{
${ }^{8}$ This follows simply from the standard result (which we do not challenge) that conditional probabilities are necessary to avoid a Dutch- Book (of the irrational kind) when bets proceed if and only if the specified condition is true. This result is applied to the extended chance-process that takes place between times $\tau$ and $\tau+3$. Peter and Mary ascertain that the draw component of this composite process produced at least one tail if and only if it is true that the whole process produced the "Tail"-utterance outcome.
} 
Table 2.

Mary's Winnings: subsidiary counter-example.

\begin{tabular}{cccccc}
\hline \multicolumn{5}{c}{ Bet 1:T( 2/3) } & \multicolumn{3}{c}{ Bet 2: HH/“Head” (1/2) } \\
\hline Outcome & Loss & Gain & Loss & Gain & Net Gain \\
\hline$H H$ & $\$ 2$ & $\$ 0$ & $\$ 3$ & $\$ 6$ & $\$ 1$ \\
$T T$ & $\$ 2$ & $\$ 3$ & $\$ 3$ & $\$ 3$ & $\$ 1$ \\
$H T$ & $\$ 2$ & $\$ 3$ & $\$ 3$ & $\$ 3$ & $\$ 1$ \\
\hline
\end{tabular}

\section{Generalizing the Counter-Example}

In the discussion above, we presumed that Peter (and Mary) knew how Paul was going to decide how to make his $(\tau+$ 3 )-utterance, but such an assumption was not critical to our revelation of the Dutch-Book. I now outline (in very brief summary) an argument that shows this, before we move on to a far more general situation.

Imagine indeed that information about the initial draw is released by some procedure quite different to that used by Paul above. But let us, for the moment, agree that Paul still informs Peter and Mary of his procedure in advance of the placement of bets; and for simplicity, let us require also that this procedure includes a guarantee to utter exactly one of "Head" or "Tail". If Peter still uses standard conditional probabilities for epistemic conditional bets, Mary can always construct a Dutch-Book exploiting Peter's choice of betting ratios.

It is well-known that Peter can be quickly Dutch-booked (without recourse to conditional bets) if the probabilities he allocates to the two utterances do not total to 1 , so at least one of the utterances can be presumed to have been allocated a probability less than $2 / 3$, say $1 / \alpha$ with $\alpha>3 / 2$ (This apparently odd choice of $\alpha$ simplifies the arithmetic below). Suppose (for the moment) that this applies to the "Tail"-utterance. All Mary has to do to get her Dutch-Book is alter the stakes in the bets comprising the principal counter-example above. A quick (but slightly untidy) calculation shows that if she makes the stake on the first bet $2 \alpha /(2 \alpha-3)$, that on the second bet $3 \alpha /(2 \alpha-3)$, and that on the third bet 1 less than that on the first bet, her winnings will always be 1. i.e. the book will be Dutch. An analogous argument applies when only the "Head"utterance has a probability less than $2 / 3$.

But suppose now that Peter is not told how Paul is to choose whether to utter "Head" or "Tail". Our gambler is faced with a situation that commonly occurs in everyday life, where information reaches us without our fully understanding how it came. If Peter is still willing to set betting ratios for Paul's utterances, exactly the same Dutch-Book strategy continues to remain available to Mary, of course. For the "objective accuracy" (etc.) of Peter's $\pi$ ("Tail") did not play any role in generating our books.

As observed above, avoidance of a Dutch-Book requires that Peter use (for bet 2) the rather different conditional probability, $\Pi\left(X \& Y^{*}\right) / \Pi\left(Y^{*}\right)$, where $Y^{*}$ means " $Y$ has been ascertained", and $\Pi(W)$ is the probability Peter allocates to each outcome $W$ of that more extensive chance-process which supplements the original chance activity with the subsequent observational process (We use capital " $P$ " and " $\Pi$ " here, to distinguish general probabilities from our concrete example, where we used lower case " $p$ " and " $\pi$ ").

\section{An Extension to Jeffrey Conditioning: "Evidential" Conditional Bets}

Imagine now that the lighting is to be dimmed between times $\tau+2$ and $\tau+3$, so that Paul will not then get a really good look at the coin, and is, he warns, likely to make an error in his identification of the face that is showing. Suppose however Paul agrees to utter "Tail" when what he sees seems more like a tail than a head. Then if Peter hears Paul utter "Tail" he is not going to acquire knowledge that the outcome $T$ has been achieved (and vice versa for "Head" and $H$ ). Under such circumstances, there is no temptation to use standard conditional probabilities for conditional bets, since the circumstances that are alleged to allow their use do not obtain.

Jeffrey however, has proposed an alternative probability that might seem to be applicable here. For he suggests that if the "Tail" utterance leaves Peter $\lambda \%$ certain that Paul has seen a tail, and $\mu \%$ certain that Paul has seen a head (where $\lambda+\mu=100$ ), then, at time $\tau+4$, Peter should update his confidence that the coin has two tails (say) from the prior value $p(T T)=33.333 \ldots \%$ to the posterior percentage $\lambda p(T T / T)+\mu p(T T / H)$. Though Jeffrey articulates his revised rule in contexts that focus primarily on diachronic processes (where gamblers place different bets at different times), the rule makes sense in our more modest context. So we can ask an obvious question: is it wise for Peter to use Jeffrey-conditionalization for his conditional bets in the circumstances sketched above, where the information released by Paul at time $\tau+3$ no longer provides knowledge that an outcome has been achieved? Such conditional bets are no longer "epistemic" then - in the sense contrasted with ontic bets above - and we will here call them "evidential" since they continue if and only if an agreed piece of evidence is obtained. If he wishes to avoid a Dutch-Book, is it sufficient for Peter to use Jeffrey-conditionalization when placing an evidentially conditional bet?

The answer to this question should be immediately clear: it is definitely "NO"! For if the Jeffrey-conditionalization was truly reliable here, it would have to work in the extreme cases, when one of $\mu$ or $\lambda$ was zero. But we have already seen that this is not so. For in such circumstances, Jeffrey's extended conditional probability (at least that version of it articulated above) simply collapses to the standard conditional probability. And by now, we have repeatedly seen that the latter is enough to allow Mary to construct Dutch-Books against Peter.

Furthermore, this form of Jeffrey's extended rule becomes totally redundant - for, as I will soon show, the conditional probability $\Pi\left(X \& Y^{*}\right) / \Pi\left(Y^{*}\right)$ can in fact accommodate the situations envisaged by Jeffrey, while the Jeffrey rule allows a Dutch-Book even when both $\mu$ and $\lambda$ are non-zero. Indeed, while it is true that the focus of this essay has until now been articulated in terms of conditions that involved Peter being supplied with knowledge (with some outcome of the primary chance-process being reliably verified), that has in fact been something of a red herring. It was there primarily because it was required to make sense of the negative claim - the claim about standard conditional probabilities that we have rejected here. But if we focus on the alternative positive result-the claim that $\Pi\left(X \& Y^{*}\right) / \Pi\left(Y^{*}\right)$ is required to avoid a Dutch-Bookthen a little reflection will indicate that there is no necessity at all for $Y^{*}$ to be restricted to the narrow meanings given it above. It can readily embrace evidential betting as well.

Indeed, we can easily generate an extremely general condi-

${ }^{9}$ See, e.g., Jeffrey, 1983: pp. 165-166. 
tional betting situation by imagining two different chance processes: process 1 that produces outcomes referred to here using early letters of the alphabet, $A, B, C, \ldots$ etc.; and process 2 that produces outcomes referred to here using late letters, $Z, Y, X, \ldots$ etc. Peter does not presume these processes to be statistically independent, so allocates joint probabilities $\Pi(A \& Z)$ to the outcomes $A$ and $Z$..., etc.; and we use $\Pi(A)$ as an abbreviation for $\Pi\left(A \& \Omega_{2}\right)$ where $\Omega_{2}$ is the universal outcome for process 2 ; $\Pi(Z)$ is similarly defined in the obvious analogous fashion.

We can now ask the question what probabilities should be used for bets on the outcomes of process 1, if those bets are conditional, and proceed if and only if process 2 produces some outcome $Y$ ? Suppose that Peter is willing to accept the betting ratio $1 / \alpha$ for a conditional bet on $B$ that proceeds if and only if $Y$ is produced. We show that if $\alpha$ is not $\Pi(Y) / \Pi(B \& Y)$, then again a Dutch-Book can be constructed.

We ignore the cases when Peter makes either $\Pi(B \& Y)$ or $\Pi(Y)$ zero, for the relevant bets will not be placed if Peter does not believe the condition will be met, or believes that $B$ is incompatible with the condition. So we can make $\beta \equiv 1 / \Pi(B \& Y)$ and $\gamma \equiv 1 / \Pi(Y)$, and consider the following book:

Bet 1: Mary bets stake $x$ on $B$ but conditionally upon $Y$. i.e. she pays Peter $x$, and in return:

- $\quad$ Peter pays her $\alpha x$ if both $B$ and $Y$ are achieved. Peter pays her nothing if $Y$ is achieved but not $B$. Peter returns her $x$ if $Y$ is not achieved.

Bet 2: Mary bets stake $y$ on $B \& Y$, unconditionally. i.e. she pays Peter $y$, and in return:

Peter pays her $\beta y$ if both $B$ and $Y$ are achieved. Peter pays her nothing if either $B$ or $Y$ is not achieved.

Bet 3: Mary bets stake $z$ on outcome $Y$, unconditionally. i.e. she pays Peter $z$, and in return: Peter pays her $\gamma z$ if $Y$ is achieved. Peter pays her nothing otherwise.

Then Mary's net winnings, in the four possible situations, viz. $B \& Y,-B \& Y, B \&-Y,-B \&-Y$, (using $-B$ for "not $B$ ". ..) are: $(\alpha-1) x+(\beta-1) y+(\gamma-1) z \quad$ when $B \& Y$ is achieved $-x-y+(\gamma-1) z$ $-y-z$ when $-B \& Y$ is achieved for either $B \&-Y$ or $-B \&-Y$.

Now, if $\beta \neq \alpha \gamma$, Mary can choose: $x=\gamma \beta /(\alpha \gamma-\beta)$ $y=-\alpha \gamma /(\alpha \gamma-\beta)$ and $z=\beta /(\alpha \gamma-\beta)$, in which case all these net winnings turn out to be +1 . (This is easy to check.) So Mary has a Dutch-Book whenever $\alpha \neq \beta / \gamma$. But $\alpha=\beta / \gamma$ if and only if $\Pi(B \& Y) / \Pi(Y)$ is the rate accepted by Peter for the conditional bet. In other words, Peter can be Dutch-Booked if he does not use this rate. This is the very general result promised above.

Superficially, this result might seem to be little more than the standard result for ontic conditional bets, but, despite the misleading similarity in expression, the result here is very different, and far broader. The standard result is in fact an extremely particular case of this general result, the particular case that presumes process 1 to be identical to process 2 . The result pursued in this paper for epistemic conditional bets is similarly a particular case of this more general result, that particular case when a) process 2 takes place after process 1 , and b) consists of the release of knowledge about the outcome of process 1 . The evidential case is the case where again process 2 takes place after process 1 , where process 2 again involves the inspection of the outcome of process 1 , but where that inspection produces outcomes that are experiences which fall short of supplying knowledge about the outcomes of process 1 .

Evidential conditional bets do not then require a special new rule, but fit exactly the same generalized pattern as the ontic and epistemic bets. Worse, we have seen that the particular rule proposed by Jeffrey does in fact allow Mary to construct some Dutch-Books. Whatever (dubious) virtues it might offer for diachronic betting, it has nothing to offer for the synchronic bets at issue here.

To give a concrete illustration of this claim that evidential betting requires no special treatment, let us return to the example introduced above (at the beginning of this discussion of Jeffrey-conditioning), when the need for some expansion of the rule for Peter's choice of betting ratio was suggested by the introduction of poor lighting. Paul's "Tail"-utterance then ceased to guarantee that there was a tail on the coin that had been drawn at time $\tau$, so bets conditional upon the "Tail"-utterance no longer qualify as "epistemic" (in the sense contrasted with ontic bets above). But we now know that Peter can be Dutch-Booked if he accepts a bet on TT that is conditional upon the "Tail"-utterance, at a rate that is not $p(T T \&$ "Tail" $) / p$ ("Tail").

Peter can calculate this safe betting ratio from his assessments of the probabilities that each of a tail and a head will be identified as a Tail. Suppose (to be concrete) that he assesses these as $75 \%$ and $15 \%$ respectively. Then he will assess $p$ ("Tail"), the probability of the "Tail"-utterance, as $1 / 2(75 \%$ $+15 \%$ ) or $45 \%$. Similarly $p$ (TT\&"Tail") will be $75 \%$ of $1 / 3$, or $25 \%$. So his safe betting ratio (for the conditional bet on $T T$, that proceeds if and only if "Tail" is uttered) in these circumstances is $25 \% / 45 \%$ or $5 / 9 \approx 55.555 \ldots \%$.

To perform this calculation, Peter needed slightly more data than is required to apply Jeffrey's rule, which only used the probability Peter would allocated to a tail if Paul made the "Tail"-utterance. The data required for Jeffrey's rule can be calculated from that required for our rule, but not vice-versa, so Jeffrey's rule is more frugal than ours. Ours however is more secure: every departure from it generates Dutch-Books.

In the example just given, a "Tail"-utterance leaves Peter $75 / 90$ certain that the coin has a tail on it, while a "Head"utterance leaves him 85/110 certain that the coin has a head on it. So according to Jeffrey's rule, the updated probability for two tails after hearing the "Tail"-utterance, will be 75/180= $5 / 12 \approx 41.666 \ldots \%$. This differs considerably from our $55.555 \ldots \%$, but, if used by Peter, allows Mary to set up the following Dutch-Book:

Bet 1: Mary bets stake $\$ 20$ on $T T$ but conditionally upon the "Tail"-utterance.

i.e. she pays Peter $\$ 20$, and in return:

- Peter pays her $\$ 48$ if "Tail" is uttered and TT is revealed at time $\tau+5$, for by hypothesis the betting ratio here is the Jeffrey-conditional $5 / 12$.

- $\quad$ Peter pays her $\$ 0$ if "Tail" is uttered but $T T$ is not revealed.

Peter returns her $\$ 20$ otherwise.

Bet 2: Peter bets stake $\$ 12$ on TT\&"Tail”, unconditionally. i.e. he pays Mary $\$ 12$; and in return:

- Mary pays Peter $\$ 48$ if "Tail" is uttered and TT is revealed at time $\tau+5$, for Peter's rate here is $1 / 4$.

- Mary pays Peter nothing if neither "Tail" is uttered nor $T T$ is revealed.

Bet 3: Mary bets stake $\$ 9$ on the "Tail"-utterance. 


\section{K. HUTCHISON}

i.e. she pays Peter \$9; and in return:

- Peter pays her $\$ 20$ if "Tail" is uttered, for Peter's betting ratio here is now $45 \%$ or $9 / 20$.

Peter pays her nothing otherwise.

Mary will now make a profit of $\$ 3$ no matter what outcome was produced in the original draw, or what utterance Paul makes at $\tau+3$. This is clear in the tabulation of her earnings in Table 3 below.

\section{An Inconclusive Conclusion: The Search for a Converse}

Having concluded, in the most general of our cases above, that Peter can be Dutch-Booked if he accepts bets on an outcome $B$ of process 1 that are conditional on the outcome $Y$ of process 2 at any rate other than $\Pi(B \& Y) / \Pi(Y)$, one is tempted to ask if some reasonable converse of this result exists. Can we argue that Peter is safe if he does use such a betting rate for each of his conditional bets?

Such a general result, however, remains in doubt, for it certainly fails with the characterizations of a Dutch-Book that dominate the literature, those that do not explicitly embrace the distinction (of p. 2) between weak and stronger books. For although all the books that have faced Peter in the examples above exploited seemingly irrational betting ratios, that is not the only way Peter can face a sure loss. Simple error can do this too. If Peter allocated subjective probabilities sufficiently in compatible with such objective realities as the real contents of

Table 3.

Mary's winnings: counter-example to Jeffrey rule.

\begin{tabular}{cccccccc}
\hline & $\begin{array}{c}\text { Bet 1: TT/"Tail” } \\
(5 / 12)\end{array}$ & $\begin{array}{c}\text { Bet 2: TT\&“Tail” } \\
(1 / 4)\end{array}$ & \multicolumn{2}{c}{$\begin{array}{c}\text { Bet 3: “Tail” } \\
(9 / 20)\end{array}$} \\
\hline Outcome & Loss & Gain & Loss & Gain & Loss & Gain & Net Gain \\
\hline HH\&“Tail" & $\$ 20$ & $\$ 0$ & $\$ 0$ & $\$ 12$ & $\$ 9$ & $\$ 20$ & $\$ 3$ \\
HH\&"Head" & $\$ 20$ & $\$ 20$ & $\$ 0$ & $\$ 12$ & $\$ 9$ & $\$ 0$ & $\$ 3$ \\
TT\&"Tail" & $\$ 20$ & $\$ 48$ & $\$ 48$ & $\$ 12$ & $\$ 9$ & $\$ 20$ & $\$ 3$ \\
TT\&"Head" & $\$ 20$ & $\$ 20$ & $\$ 0$ & $\$ 12$ & $\$ 9$ & $\$ 0$ & $\$ 3$ \\
HT\&"Tail”, & $\$ 20$ & $\$ 0$ & $\$ 0$ & $\$ 12$ & $\$ 9$ & $\$ 20$ & $\$ 3$ \\
HT\&"Head" & $\$ 20$ & $\$ 20$ & $\$ 0$ & $\$ 12$ & $\$ 9$ & $\$ 0$ & $\$ 3$ \\
\hline
\end{tabular}

the urn or Paul's actual epistemic procedure, he becomes exposed to (weak) Dutch books. So avoidance of a sure loss requires Peter to do more than just allocate an appropriate conditional probability. And it is surely impossible to establish that Peter's avoidance of one particular irrational bet protects him from all others, right through his life.

Yet some partial converses must surely hold, since we all do believe that Peter can sometimes make conditional bets without being Dutch-Booked. But it remains unclear what restrictions need to be placed on such converses to make them valid. A converse claim restricted to stronger books could well survive, but any such claim would have to be carefully formulated, and I do not know of any claims that are clearly valid.

\section{REFERENCES}

De Finetti, B. (1974-1975). Theory of probability: A critical introductory treatment. (2 vols.) A. Machí, \& A. Smith (Trans.), New York: Wiley.

Gillies, D. (2000). Philosophical theories of probability. London: Routledge.

Hacking, I. (1967). Slightly more realistic personal probability. Philosophy of Science, 34, 311-325. doi:10.1086/288169

Harman, G. (1983). Problems with probabilistic semantics. In A. Orenstein \& R. Stern (Eds.), Developments in semantics (pp. 242-245). New York: Haven.

Howson, C. (1977). Bayesian rules of updating. Erkenntnis, 45, $195-$ 208.

Howson, C., \& Urbach, P. (2006). Scientific reasoning: The Bayesian approach (3rd ed.). Chicago, IL: Open Court.

Hutchison, K. (1999). What are conditional probabilities conditional upon? British Journal for the Philosophy of Science, 50, 665-695. doi:10.1093/bjps/50.4.665

Jeffrey, R. (1983). The logic of decision (2nd ed.). Chicago, IL: University of Chicago Press.

Skyrms, B. (1987). Dynamic coherence and probability kinematics. Philosophy of Science, 54, 1-20. doi:10.1086/289350

Talbott, W. (2008). Bayesian epistemology (revision of Mar 26, 2008). Stanford Encyclopedia of Philosophy. URL (last checked 9 Jun 2012). http://plato.stanford.edu/entries/epistemology-bayesian

Teller, P. (1973). Conditionalization and observation. Synthese, 26, 218-258. doi:10.1007/BF00873264

Weatherson, B. (2003). From classical to intuitionistic probability, Notre Dame Journal of Formal Logic, 44, 111-123. doi:10.1305/ndjfl/1082637807. 